\title{
AN EXTREMAL CONTROL IN DIFBERENTIAL GAMES
}

\author{
A.G. Chentsov \\ Institute of Mathematics and Mechanics, \\ Ural Scientific Center of the Academy of \\ Sciences of the USSR \\ S. Kovalevskaja st. 16, Sverdlovsk, USSR
}

Let us consider a system

$\frac{d x}{d t}=f(t, x, u, v) ; x \in R^{n}, u \in P \subset R^{p}, v \in Q \subset R^{q}$.

Here $P$ and $Q$ are compact sets, $f(\cdot)$ is a continuous function, continuously differentiable in $\boldsymbol{X}$. It is assumed that the following condition of uniform extendability of the solutions holds: for any bounded set $K \subset R^{n}$ there exists $\beta=\beta(K)>0$ such that any solution $x(t)=x\left(t, t_{*}, x_{*}\right)$ of the equation

$$
\frac{d x}{d t} \in \overline{c o}\{f: f=f(t, x, u, v), u \in P, v \in Q\}
$$

with the initial condition

$$
t_{*} \in\left[t_{0}, g\right], x_{*} \in K \text { is }
$$

uniformly bounded in the segment $\left[t_{*}, \boldsymbol{v}\right]:\|x(t)\| \leqslant \beta$. Here the symbol $\|\cdot\|$ denotes Euclidean norm.

There is given a function $\omega(x, m)$ defined on the space $R^{n} \times M_{\text {whe- }}$ reM is a compact subset of $R^{m}$, which is continuous and continuously differentiable in $x$ on domain $\omega \in\left(\omega_{0}, \omega^{\circ}\right), \omega_{0}<\omega^{\circ}$. The first player by choosing a control $u \in P$, tries to minimize the value of the functional $\rho_{M}(x[v])=\min _{M} \omega(x[v], m)$. The second player by choosing a control tries to maximize it.

Similarly to [1], any upper semicontinuous with respect to inclusion in $(t, x)$ function $U(t, x) \subset P$ will be called an admissible strategy $U$ of the first player. The admissible strategy $V$ of the second player can be defined similarly; any upper semicontinuous with respect to inclusion in $(t, x, v)$ function $U_{v}(t, x, v) c P$ will be called a counterstrategy $U_{v}$ of the first player. We shall say that a function $x[t]$ is the motion generated by a strategyll 
441

(counterstrategy $U_{v}$ ) if it is a solution of the equation

$$
\begin{aligned}
& \frac{d x}{d t} \in \overline{c o}\{f: f=f(t, x, u, v) ; u \in U(t, x), v \in Q\} \\
& x\left[t_{0}\right]=x_{0} ; \\
& \frac{d x}{d t} \in \overline{c o}\left\{f: f=f(t, x, u, v) ; u \in U_{v}(t, x, v), v \in Q\right\} \\
& x\left[t_{0}\right]=x_{0} .
\end{aligned}
$$

Any solution of the equation

$$
\begin{aligned}
\frac{d x}{d t} & \in \overrightarrow{c o}\{f: f=f(t, x, u, v) ; u \in U(t, x), v \in V(t, x)\} \\
& x[t, 0]=x_{0}
\end{aligned}
$$

will be called the motion generated by a pair of strategies $(U, V)$.

Problem I. There is required to build an optimal counterstrategy $\mathrm{U}_{\mathrm{v}}^{0}$ such that:

$$
\begin{aligned}
& \max _{\left\{x[\cdot] \mid U_{v}^{0}\right\}} \min _{M} \omega(x[v], m)=\min _{\left\{U_{v}\right\}} \max _{\left\{x[\cdot] \mid U_{v}\right\}} \min _{M} \omega(x[v] m) \\
& \text { And if the condition } \\
& \min _{P} \max _{Q} s^{\prime} f(t, x, u, v)=\max _{Q} \min _{P} s^{\prime} f(t, x, u, v)(2)
\end{aligned}
$$

holds for every $s \in R^{n}, t \in[t, q], x \in R^{n}$ it is required to find a solution in the class of admissible strategies.

Problem 2. It is required to find a pair $\left(\mathrm{U}^{\circ}, \mathrm{V}^{\circ}\right)$ of optimal strategies which satisfies

$$
\begin{aligned}
& \min \max \min \omega(x[v], m)= \\
& \{U\} \quad\{x[\cdot] \mid U\} \quad M \\
& =\max \quad \min \omega(x[v], m)= \\
& \left\{x[\cdot] \mid U^{\circ}\right\} \quad M \\
& =\min _{\left\{x[\cdot] \mid V^{\circ}\right\}} \min _{M} \omega(x[\downarrow], m)= \\
& =\max \min \min \omega(x[v], m) \text {. } \\
& \{V\} \quad\{x[\cdot] \mid V\} \quad M
\end{aligned}
$$


Let us call a set of all regular Borel measures (r. B.m.) Dू( on $\left[t_{*}, \mathfrak{q}\right] \times \mathrm{P} \times \mathrm{Q}$, having Lebesgue projection $[2,3]$ on $[t, q]$, a class $\left\{H\left(m(\cdot),\left[t_{*}, \mathbb{J}\right]\right\}\right.$ of admissible open$-100 p$ controls of the first player on the segment $\left[t_{*}, \mathfrak{V}\right]$. A set of all r.B.m. $v(\cdot)$ on $[t,, \mathbb{J}] \times Q$ having Lebesgue projection on $\left[t_{*}, \mathcal{Y}\right]$ will be called a class $\left\{E(M(\cdot)),\left[t_{*}, j\right]\right\}$ of admissible open -loop controls of the second player on the segment $\left[t_{*}, \mathbb{\$}\right]$. The set of all controls $\mathfrak{Q}(\cdot) \in\left\{H\left(m(\cdot),\left[t_{*}, \mathfrak{s}\right]\right\}\right.$ coordinated $[2,3]$ with open-loop control $O($.$) of the second player we shall call a$ program $\{\Pi(\nu(\cdot)),[t *, \$]\}$.

To every open-1oop control can be put in correspondence a program motion $[2,3] \varphi\left(\cdot, t_{*}, x_{*}, 2(\cdot)\right)$ with initial condition $\left(t_{*} x_{*}\right)$. let $G\left(q_{*}, t_{*}, x_{*}, V(\cdot)\right)$ be a set of attainability $[1,3]$ for a program $\{\Pi(v(\cdot),[t *, j]\}$ at the moment $q$

$$
\varepsilon^{0}\left(t_{*}, x_{*}\right)=\max _{\{E(m(\cdot)),[t, q]\}} \min _{G\left(v_{,}, t_{*}, x_{*}, v(.)\right)} \min _{M} \omega(x, m)
$$

open-loop controls giving minimum and maximum ( 3 ) will be called optimal ones.

Theorem I. Iet the following condition hold for a position $\left(t_{*}, x_{*}\right)$ and open-loop control $\nu^{*}(\cdot) \in\left\{E(m(\cdot)),\left[t_{*}, j\right]\right\}$ :

\section{min}

$G\left(S, t *, x *, v^{*}(\cdot)\right)$

$$
\min _{M} \omega(x, m) \in\left(\omega_{0}, \omega^{\circ}\right) \text {. }
$$

Then the optimal open-loop control $\eta^{\circ}(\cdot)$ in the program $\left\{\prod\left(\nu^{*}(\cdot)\right)\left[t_{*}, g\right]\right\}$ satisfies the minimum principle

$$
\begin{aligned}
& \iint_{P} \int_{Q} s_{0}^{\prime}(t) f\left(t, \varphi^{\circ}(t), u, v\right) v^{\circ}(d t \times d u \times d v)= \\
& =\int_{\Delta} \int_{Q} \min _{P} s_{0}^{\prime}(t) f\left(t, \varphi^{\circ}(t), u, v\right) v^{*}(d t \times d v)
\end{aligned}
$$

where $\Delta$ is an arbitrary Borel subset of $\left[t_{*}, \mathfrak{Q}\right]$,

$$
\begin{aligned}
& \varphi^{\circ}(t)=\varphi\left(t, t *, x_{*}, 2^{\circ}(\cdot)\right), \\
& s_{0}^{\prime}(t)=\left[\frac{\partial}{\partial x} \omega\left(\varphi^{\circ}(v), m^{\circ}\right)\right]^{\prime} S\left(q, t, \varphi^{\circ}(), 2^{0}(\cdot),\right.
\end{aligned}
$$

$m$ gives a minimum to $n 2 i n, \omega\left(\varphi^{\circ}(\mathcal{J}), m\right), S\left(J, t, \varphi^{\circ}(\cdot), \eta^{\circ}(\cdot)\right.$ )is fundamental solution matrix for a corresponding system in variations.

Theorem 2. Let $E^{0}\left(t_{*}, x_{*}\right) \in\left(\omega_{0}, \omega^{\circ}\right)$ and an open-loop control $v^{\circ}(.) \in\left\{E(m(.)),\left[t_{*}, \vartheta^{*}\right]\right\}_{\text {opt }}$ be such that an optimal control $\underline{D}^{\circ}(\cdot)$ in the program $\left\{\Pi\left(\gamma^{\circ}(\cdot),\left[t_{*}, \mathfrak{J}\right]\right\}\right.$ be unique, moreover a point $m^{\circ} \in M$, giving $\min M \omega\left(\varphi^{\circ}(\delta), m\right)$, be also unique. Then for arbitrary Borel subset $\triangle$ 
443

$$
\begin{aligned}
& \iint_{\Delta} \int_{Q} s_{0}^{\prime}(t) f\left(t, \varphi^{\circ}(t), u, v\right) \eta^{\circ}(d t \times d u x d v)= \\
& =\int_{\Delta} \max _{Q} \min _{P} s_{0}^{\prime}(t) f\left(t, \varphi^{\circ}(t), u, v\right) m(d t) .
\end{aligned}
$$

Here notations $\varphi^{\circ}(t), s_{0}(t)$ have the same meaning as in theorem 1.

For every position $\left(t_{*}, x_{*}\right), \varepsilon^{0}\left(t_{*}, x_{*}\right) \in\left(\omega, \omega^{0}\right)$, de note by $S_{0}\left(t_{*}, x_{*}\right)$ a set of all vectors $s_{0}$ of the form

$$
s_{0}^{\prime}=\left[\frac{\partial}{\partial x} \omega\left(\varphi_{0}(v), m_{0}\right)\right]^{\prime} S\left(\vartheta, t_{*}, \varphi_{0}(\cdot), \eta_{0}(\cdot)\right),
$$

where $\varphi_{0}(\cdot)=\varphi\left(\cdot, t_{*}, x_{*}, \eta_{0}(\cdot)\right), \eta_{0}(\cdot) \in\left\{\Pi\left(\nu_{0}(\cdot)\right),\left[t_{*}, v^{2}\right]\right\}$ $\left(\eta^{0}(\cdot), \nu_{0}(\cdot)\right.$ are optimal for the position $\left(t_{*}, x_{*}\right), m_{0}$ gives $\min _{M} \omega\left(\varphi_{0}(v), m_{0}\right)$. It will be said that a game is perfectly regular if the set $S_{0}\left(t_{*}, x_{*}\right)$ consists of only one vector $s_{0}=s_{0}\left(t_{*}, x_{*}\right)$ for every position $\left(t_{*}, x_{*}\right), \varepsilon^{0}\left(t_{*}, x_{*}\right) \in\left(\omega_{0}, \omega^{0}\right)$.

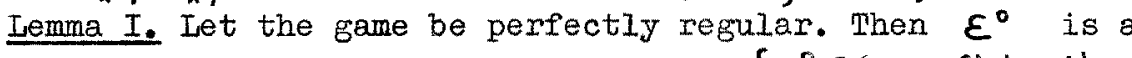
continuously differentiable function in domain $\left\{\varepsilon^{\circ} \in\left(\omega_{0}, \omega^{\circ}\right), t \in\left(t_{0},{ }^{0}\right)\right\}$ and

$$
\left.\frac{\partial \varepsilon^{0}}{\partial x}\right|_{(t, x)}=s_{0}(t, x),\left.\frac{\partial \varepsilon^{0}}{\partial t}\right|_{(t, x)}=-\max _{Q} \min _{P} s_{0}^{\prime}(t, x) f .
$$

Extremal strategies under the condition of perfect regularity on domain of differentiability are defined by the relations :

$$
\begin{aligned}
& U^{0}\left(t_{*}, x_{*}\right)=\left\{u^{0}: u^{0} \in P, \max _{Q} s_{0}^{\prime}\left(t_{*}, x_{*}\right) f\left(t_{*},\right.\right. \\
& \left.\left.x_{*}, u^{0}, v\right)=\min _{P} \max _{Q}^{\prime}\left(t_{*}, x_{*}\right) f\left(t_{*}, x_{*}, u, v\right)\right\}, \\
& V^{\circ}\left(t_{*}, x_{*}\right)=\left\{v^{0}: v^{\circ} \in Q, \min _{P} s_{0}^{\prime}\left(t_{*}, x_{*}\right) f\left(t_{*},\right.\right. \\
& \left.\left.x_{*}, u, v^{0}\right)=\max _{Q} \min _{P} s_{0}^{\prime}\left(t_{*}, x_{*}\right) f\left(t_{*}, x_{*}, u, v\right)\right\}, \\
& U_{v}^{0}\left(t_{*}, x_{*}, v\right)=\left\{u^{0}: u^{0} \in P, s_{0}^{\prime}\left(t_{*}, x * f f\left(t_{*}\right.\right.\right. \\
& \left.\left.x_{*}, u^{0}, v\right)=\min _{P} s_{0}^{\prime}\left(t_{*}, x_{*}\right) f\left(t_{*}, x_{*}, u, v\right)\right\}
\end{aligned}
$$


Theorem 3. Let the game be perfectly regular and $\varepsilon^{\circ}\left(t_{0}\right.$, $\left.x_{0}\right) \in\left[\omega_{0}, \omega^{0}\right)$. Then a counterstrategy $U_{v}^{0}$ (and under condition (2) strategy $U^{\circ}$ ) solves the problera 1. If (2) holds and $\varepsilon^{\circ}\left(t_{0}, x_{0}\right) \in\left(\omega_{0}, \omega^{0}\right)$, a pair $\left(U^{0}, V^{0}\right)$ solves the problem 2 and $\varepsilon^{\circ}\left(t_{0}, x_{0}\right)$ is the cost of the positional game in pure strategies.

For every optimal for the position $\left(t_{*}, x_{*}\right)$ open-loop control $V_{0}(\cdot)$ of the second player let us find a set $S_{0}\left(t_{*}, x_{*}, \nu_{0}(\cdot)\right)$ of all vectors $s_{0}$ determined by (4) with fixed control $\nu_{0}(\cdot)$. Assume for every position $\left(t_{*}, x_{*}\right) \quad t_{*} \in\left(t_{0}, \vartheta\right)$ and $\varepsilon^{0} \in\left(\omega_{0}, \omega^{0}\right) \quad$ for every optimal open-loop control $\nu_{0}(\cdot)$ of the second player the set $S_{0}\left(t_{*}, x_{*}, \nu_{0}(\cdot)\right)$ consists of the unique vector $s_{0}=s_{0}\left(t_{*}, x_{*}, \nu_{0}(\cdot)\right)$. This condition is fulfilled, for example, when

$$
f(t, x, u, v)=A(t) x+f(t, u, v),
$$

the set $M \subset R^{n} \quad$ is convex and closed, and $\omega(x, m)=\|x-m\|$. Theorem 4. Let $\varepsilon^{\circ}(t, x)$ be differentialable in the domain $\Gamma=$ $=\left\{t \in\left(t_{0}, \vartheta\right), \varepsilon^{0} \in\left(\omega_{0}, \omega^{0}\right)\right\}$. Then for every position $\left(t_{*}, x_{*}\right) \in \Gamma$ the set $S_{0}\left(t_{*}, x_{*}\right)$ consists of the unique vector $1_{0}=$ $=s_{0}\left(t_{*}, x_{*}\right)$ and the partial derivatives of $\varepsilon^{\circ}(t, x)$ satisfy $(5)$.

$$
R e f e r e n c e s
$$

1. N.N. Krasovskii. Game problems on encounter of motions. M., Nauka, 1970. (Russian).

2. V.D. Batuhtin, N.N. Krasovskii. Maximin problem for open-loop control. Izv. AN SSSR. Tehn. kibernet., 1972, Ne 6. (Russian ).

3. A.G. Chentsov. On game problem for open-loop control. Dokl. AN SSSR, 1973, 213, 2. (Russian). 